\title{
1-MCP EM MANGABAS ARMAZENADAS EM TEMPERATURA AMBIENTE E A $11^{\circ} \mathrm{C}^{1}$
}

\author{
RAQUEL PIRES CAMPOS ${ }^{2}$, BEATRIZ $\mathrm{KNOCH}^{3}$, PRISCILA AIKO HIANE ${ }^{3}$, \\ MARIA ISABEL LIMA RAMOS ${ }^{3}$, MANOEL MENDES RAMOS FILHO 3
}

RESUMO - A mangaba, fruto nativo altamente perecível, apresenta reduzida vida útil pós-colheita. Novas tecnologias de conservação de frutos têm sido desenvolvidas, e o uso de reguladores vegetais tem sido promissor. Este trabalho avaliou a aplicação do regulador de etileno1-MCP em diferentes concentrações (250; 500 e $1.000 \eta L^{-1}$ ), com o objetivo de verificar o prolongamento da vida útil dos frutos e suas características físicas e químicas durante o armazenamento em temperatura ambiente e a $11^{\circ} \mathrm{C}$. O uso $1-\mathrm{MCP}$, independentemente das três concentrações utilizadas, apresenta grande benefício na conservação pós-colheita dos frutos em condição ambiente, aumentando o período de vida útil da mangaba, assim como o uso de armazenamento a $11^{\circ} \mathrm{C}$, os quais favoreceram a redução da perda de massa e a manutenção dos teores de acidez titulável, sólidos solúveis e vitamina $\mathrm{C}$; e quando associado à aplicação de 1-MCP, reduziu os descartes por amadurecimento excessivo. Os frutos apresentaram bom conteúdo de fenóis totais, com incrementos durante o armazenamento, principalmente com uso da concentração de $1.000 \eta \mathrm{L} \mathrm{L}^{-1}$ de 1-MCP.

Termos para indexação: conservação, fenóis totais,Hancornia speciosa, pós-colheita.

\section{1-MCP ON MANGABA STORED AT AMBIENAL TEMPERATURE AND $11^{\circ} \mathrm{C}$}

\begin{abstract}
The mangaba, a highly perishable native fruit, presents reduced shelf-life. New technologies of fruit preserving have been developed and the use of plant growth regulators has been promising. This study evaluated the application of the regulator ethylene 1-MCP at different concentrations $(250,500$ and $1000 \eta \mathrm{L} \mathrm{L}^{-1}$ ), in order to verify the increase of fruits durability and their physical and chemical characteristics during storage at room temperature and at $11^{\circ} \mathrm{C}$. The use of $1-\mathrm{MCP}$, at every concentrations used, was beneficial to the fruits post-harvest under natural conditions, increasing the shelf life of mangaba, and so was the use of storage at $11^{\circ} \mathrm{C}$, which favored the reduction of weight loss and the maintenance of the levels of acidity, soluble solids and vitamin C. When associated with the application of 1-MCP, the loss by excessive maturation decreased. The fruits presented a proper content of total phenols, which increased during storage, mainly under the concentration of $1.000 \eta \mathrm{L} \mathrm{L}^{-1}$ of $1-\mathrm{MCP}$.
\end{abstract}

Index Terms: conservation, Hancornia speciosa, post harvest, total phenols.

\section{INTRODUÇÃO}

Nativa do Brasil, a mangabeira (Hancornia speciosa Gomes) pertence à família das apocináceas, é planta arbórea de porte médio e atinge de 5 a 10 metros de altura (EMBRAPA, 2007). Possui vasta ocorrência no bioma Cerrado, assim como em outras regiões, ocorre em agrupamentos naturais, facilitando ações de extrativismo e conservação (LORENZI, 2009). Com potencial para o aproveitamento variado, seus frutos apresentam valor comercial significativo (SOARES et al., 2005). Seu maior uso é por meio de processamento de polpa congelada, suco engarrafado, sorvete, doce, licor e geleia, xarope, compotas, vinho, vinagre, além do consumo in natura (EMBRAPA, 2007).

$\mathrm{O}$ consumo de mangaba oferece benefícios à saúde através do fornecimento elevado de antioxidantes naturais (ALMEIDA et al., 2011). A mangabeira produz frutos aromáticos, delicados, saborosos e

\footnotetext{
${ }^{1}$ Trabalho Sinfruit 179 - Simpósio Internacional de Fruticultura - Avanços na Fruticultura (17 a 21 Outubro)

${ }^{2}$ DCR em Tecnologia pós-colheita (Fundect/CNPq/UFMS). e-mail: raquel.campos@ufms.br

${ }^{3}$ Prof. Dr. do Departamento de Tecnologia de Alimentos e Saúde Pública, Universidade Federal de Mato Grosso do Sul (UFMS), Caixa Postal 549, CEP 79070-900, Campo Grande-MS. e-mails: priscila.hiane@ufms.br; maria-isabel.ramos@ufms.br; manoel. filho@ufms.br.
} 
nutritivos, com teor de proteína de 1,3 a 3,0\%, com teores de vitaminas e sais minerais superiores aos da maioria das espécies frutíferas, tendo excelente aceitação no mercado (FERREIRA, 2007). A polpa dos frutos apresenta baixo conteúdo lipídico e calórico, é fonte de fibras e de minerais (MARIN et al., 2009; SILVA et al. 2008).

Parte significativa da produção é perdida em virtude do curto período de safra, da ausência de uma coloração específica que identifique o fruto maduro e de sua alta perecibilidade, o que compromete seu escoamento e produção (EMBRAPA, 2007). As tecnologias pós-colheita fornecem perspectivas cada vez mais amplas e promissoras de atividade e agregação de renda por parte de agricultores familiares e extrativistas, aumentando o período de comercialização e reduzindo as perdas pós-colheita dos frutos nativos. A utilização de baixas temperaturas é um eficiente método de conservação pós-colheita de frutos, e o uso do 1-metilciclopropeno (1-MCP) retarda o amadurecimento mantendo a coloração verde e o frescor dos frutos. O 1-MCP é um regulador vegetal que vem sendo testado em diferentes produtos hortícolas em pós-colheita, visando a impedir a ação do etileno sobre o amadurecimento, competindo com os sítios receptores nas membranas celulares, impedindo o seu estímulo fisiológico (CHITARRA; CHITARRA, 2005).

Visando ao maior período de conservação de mangabas, avaliou-se a aplicação de diferentes concentrações de 1-MCP sobre características pós-colheita de frutos armazenados em condições ambiente e refrigerada.

\section{MATERIAL E MÉTODOS}

Os frutos foram colhidos no estágio "de vez", no município de Corguinho - MS. Foram selecionados quanto ao tamanho e ausência de danos e lavados com detergente neutro. Após secagem ao ar, os frutos foram submetidos a aplicações de 1-MCP nas concentrações $250 ; 500$ e $1.000 \eta L_{L}^{-1}$, durante $12 \mathrm{~h}$, seguidos de armazenamento à temperatura ambiente $(250 \mathrm{~A} ; 500 \mathrm{~A}$; e $1.000 \mathrm{~A})$ ou armazenamento a $10^{\circ} \mathrm{C}$ (250R; 500R e 1.000R), além dos grupos Controle Ambiente (CA) e Controle Refrigerado (CR).

O 1-MCP (SmartFresh ${ }^{\circledR}$ ) foi utilizado em aplicação única, nos frutos recém-colhidos com tempo de exposição de doze horas em condições de temperatura ambiente e embalagem hermeticamente fechada. A quantidade calculada de SmartFresh foi colocado em um recipiente plástico rígido, com $3 \mathrm{~mL}$ de água destilada e filtrada. Após agitação, a tampa foi removida no interior da caixa de isopor, a qual foi rapidamente lacrada.

Os frutos foram medidos por meio de paquímetro, obtendo-se os diâmetros longitudinal e equatorial. A avaliação de perda de massa foi realizada por meio de pesagens das parcelas, e os descartes foram considerados ao longo do período de armazenamento.

As análises químicas foram realizadas no início, meio e final do armazenamento. Foram avaliados os teores de sólidos solúveis e acidez titulável segundo metodologia do Instituto Adolfo Lutz (BRASIL, 2005). A determinação do teor de ácido ascórbico foi realizada por titulação com o reagente 2,6-diclorofenolindofenol, de acordo com metodologia proposta por Carvalho et al. (1990).

Determinações dos teores de fenóis totais foram realizadas no início e final do período de armazenamento, em triplicata. Foram preparados extratos aquosos com $5 \mathrm{~g}$ de polpa fresca e dissolvidos em metanol para uma concentração de sólidos de 6 mg.mL ${ }^{-1}$, seguindo metodologia descrita em Roesler et al. (2007), os quais foram submetidos à reação colorimétrica descrita por Swain e Hills (1959) a 760 nm, utilizando o reagente de Folin-Ciocalteau. A curva-padrão foi preparada com ácido gálico, e os resultados dos extratos, expressos como equivalentes de ácido gálico (EAG) em mg de ácido gálico por $100 \mathrm{~g}$ de massa seca.

Foi utilizado esquema fatorial com quatro tratamentos $\mathrm{x}$ duas condições de armazenamento (ambiente e refrigerado) $\mathrm{x}$ três períodos de tempo (inicial, parcial e final), com cinco frutos por parcela e quatro repetições por tratamento. Os dados foram submetidos à análise de variância (ANOVA), e para comparação das médias, foi utilizado o teste de Tuckey, em nível de $5 \%$ de probabilidade, com auxílio do programa estatístico BioEstat 5.0 (AYRES et al., 2007).

\section{RESULTADOS E DISCUSSÃO}

A vida útil das mangabas foi de nove dias em armazenamento ambiente (CA), e com a aplicação de 1-MCP estendeu-se para 15 dias, independentemente da concentração utilizada. A refrigeração prolongou a vida útil dos frutos para 24 dias de armazenamento (CR) e, quando associada com o uso de 1-MCP, prolongou para 27 dias, sem diferenças para as concentrações utilizadas. Segundo Carnelossi et al. (2004), as mangabas caídas tornaram-se deterioradas após 3 e 7 dias de armazenamento a $25^{\circ} \mathrm{C}$ e $18^{\circ} \mathrm{C}$, o que demonstra a importância da colheita no ponto de maturidade fisiológica ("de vez"), visando ao prolongamento da vida pós-colheita dos frutos, assim como o uso de refrigeração. 
Os frutos apresentaram valores médios de diâmetro longitudinal de 34,29 $\pm 2,07$ mm e diâmetro equatorial de 35,22 $\pm 1,44 \mathrm{~mm}$, dados não apresentados na forma de tabela, os quais estão próximos dos encontrados por Guilherme et al. (2007), com $37,59 \pm 10,66 \mathrm{~mm}$ e $34,53 \pm 8,83$, respectivamente, e por Soares et al. (2008), com $32 \pm 9 \mathrm{~mm}$ e $28 \pm 8$ $\mathrm{mm}$, respectivamente. A menor variação encontrada no presente trabalho deve-se a seleção dos frutos realizada na montagem do experimento.

A perda de massa dos frutos, após nove dias em armazenamento ambiente, foi de 12,57\% (CA) e foi reduzida significativamente $(p<0,05)$ com a aplicação de 1-MCP, obtendo entre 7,38\% (1.000A) e $9,21 \%(500 \mathrm{~A})$, enquanto para a perda de massa, os frutos refrigerados apresentavam em torno de 3,35\% (250 R) e 3,98\% (CR) neste período (Figura 1). Após 24 dias de armazenamento refrigerado, os frutos com aplicação de 1-MCP atingiram perda de massa acumulada de $13,22 \% ; 13,69 \% ; 15,55 \%$ e $18,65 \%$ para $250 \mathrm{R}, 500 \mathrm{R}, 1.000 \mathrm{R}$ e $\mathrm{CR}$, respectivamente, sem apresentar diferenças estatísticas entre si. Santos et al. (2009) encontraram perda de massa superior em mangabas verdes $(25,28 \%)$ após 15 dias de armazenamento a $10^{\circ} \mathrm{C}$, principalmente devido à menor umidade relativa do ar.

O valor médio da acidez titulável dos frutos recém-colhidos foi de $0,554 \pm 0,106 \mathrm{~g}$ de ácido cítrico $100 \mathrm{~g}^{-1}$ de polpa. Durante o armazenamento, os frutos apresentaram valores parciais entre $0,395{\mathrm{~g} 100 \mathrm{~g}^{-1}}^{-1}$

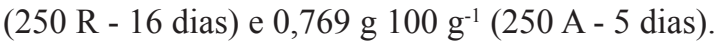
Ao final do armazenamento, o maior valor médio

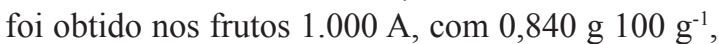
diferindo estatisticamente dos outros tratamentos, seguido pelo 500A, CA e 250A (0,714; 0,692 e 0,639 g $100 \mathrm{~g}^{-1}$, respectivamente), e os frutos refrigerados apresentaram valores inferiores e sem diferenças estatísticas entre os tratamentos $(0,574$ e $0,474 \mathrm{~g}$ $100 \mathrm{~g}^{-1}$ ) (Figura 2). Carnelossi et al. (2004) relataram o aumento da acidez titulável em mangabas com o armazenamento ambiente e refrigerado, e valores próximos do encontrado no presente trabalho, de $0,72 \%$ de ácido cítrico, assim como Soares et al. (2008) em mangabas armazenadas sob atmosfera modificada e sob refrigeração, valores de 0,38 a $0,78 \%$. Entretanto, valores superiores, acima de 1,5\%, foram encontrados em mangabas por Souza et al. (2007), Moura et al. (2002) e Santos et al. (2009).

$\mathrm{O}$ aumento da acidez nos frutos, com o amadurecimento em temperatura ambiente, deve-se à hidrólise de pectina, com liberação dos ácidos galacturônicos pela ação das enzimas pectinametilesterase e poligalacturonase (ALVES et al., 2000). A aplicação de 1-MCP, na concentração $1.000 \eta \mathrm{L}$
$\mathrm{L}^{-1}$, contribuiu para o aumento da acidez dos frutos armazenados em temperatura ambiente, devido possivelmente à redução das taxas respiratórias. Teores mais altos de acidez pelo uso do 1-MCP foram descritos para frutos climatéricos, maçãs, graviolas (respectivamente, WILLS; KU, 2002; KRAMES et al., 2001; LIMA et al., 2001 apud DOLLHOJO et al., 2009) e mangas 'Kent', possivelmente, devido à diminuição da atividade de enzimas relacionadas ao metabolismo respiratório (GARCIA ESTRADA et al., 2000 apud DOLLHOJO et al., 2009).

Os frutos apresentaram teores médios de sólidos solúveis entre 18,4 e $23,2^{\circ}$ Brix ao longo do armazenamento, sendo que os frutos dos tratamentos $500 \mathrm{R}$ e $1.000 \mathrm{R}$ atingiram os maiores valores em relação ao Controle Refrigerado parcial e final (Figura 2). Valores inferiores ao encontrado no presente trabalho foram relatados por Souza et al. (2007) com média geral de $17,23^{\circ}$ Brix; por Carnelossi et al. (2004) em mangabas mantidas à temperatura de $6^{\circ} \mathrm{C}$, com valores entre 13,1 e 15,67 ${ }^{\circ}$ Brix para frutos "de vez"; por Santos et al. (2009) entre 11 e $15^{\circ}$ Brix durante o armazenamento em ambiente e entre 11 e $18^{\circ}$ Brix a $10^{\circ} \mathrm{C}$.

O teor de vitamina $\mathrm{C}$ foi mantido durante a conservação pós-colheita das mangabas, principalmente com o uso da refrigeração, as quais apresentaram valor inicial de $132,61 \pm 21,88 \mathrm{mg}$ de ác. ascórbico $100 \mathrm{~g}^{-1}$ polpa. Os valores finais variaram entre 132,60 e $166,49 \mathrm{mg}^{100 \mathrm{~g}^{-1}}(250 \mathrm{~A}$ e $1.000 \mathrm{~A}$, respectivamente) e para os frutos armazenados sob refrigeração entre 148,78 e $161,35{\mathrm{mg} 100 \mathrm{~g}^{-1}}^{-1}$ (1.000R e CR, respectivamente) (Figura 2). Valores semelhantes foram encontrados em mangabas, 139,6 $\mathrm{mg} 100 \mathrm{~g}^{-1}$ por Alves et al. (2000) e 155,54 mg de ác. ascórbico $100 \mathrm{~g}^{-1}$ polpa por Guilherme et al. (2007). Valores superiores foram relatados por Carnelossi et

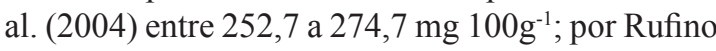

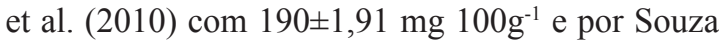
et al. (2007) em clones de mangaba com 164,77 a $188,7554 \mathrm{mg}$ de ác. ascórbico $100 \mathrm{~g}^{-1}$ polpa .

As variações nos conteúdos de acidez titulável, sólidos solúveis e vitamina $\mathrm{C}$ dos frutos, nos diferentes trabalhos de pesquisa, estão relacionadas às diferentes condições edafoclimáticas e genéticas a que as mangabeiras estão submetidas.

O conteúdo de fenóis totais inicial foi de $11,29 \mathrm{mg} \mathrm{EAG} \mathrm{g}^{-1} \mathrm{~ms}$ e aumentou com o armazenamento, atingindo valores finais de 29,04 mg EAG $\mathrm{g}^{-1} \mathrm{~ms}(1.000 \mathrm{~A})$ aos 15 dias e $23,65 \mathrm{mg} \mathrm{EAG} \mathrm{g}^{-1} \mathrm{~ms}$ (1000R) aos 27 dias (Figura 3). Valores semelhantes em mangabas maduras foram obtidos por Souza et al. (2007), com teor de compostos fenólicos em média de $0,37 \%$ e por Alves et al. (2000) com $0,29 \%, 0,31 \%$ 
e $0,33 \%$, respectivamente, para os compostos fenólicos poliméricos, oligoméricos e dímeros. Teores próximos ao encontrado inicialmente nas mangabas foram relatados por Canuto et al. (2010) em outros frutos nativos, como araçá-boi, cajá, caju, graviola e murici $\left(0,6 \mathrm{mmol} \cdot \mathrm{L}^{-1}\right.$ de ácido gálico). Vasco et al. (2008), estudando 17 frutas do Equador, relataram ampla gama de variação nos teores de compostos fenólicos associados à capacidade antioxidante, identificando três grupos de frutos contendo baixo, médio e alto teor, sendo que a mangaba poderia ser classificada como médio conteúdo de fenóis totais com base nesta classificação.
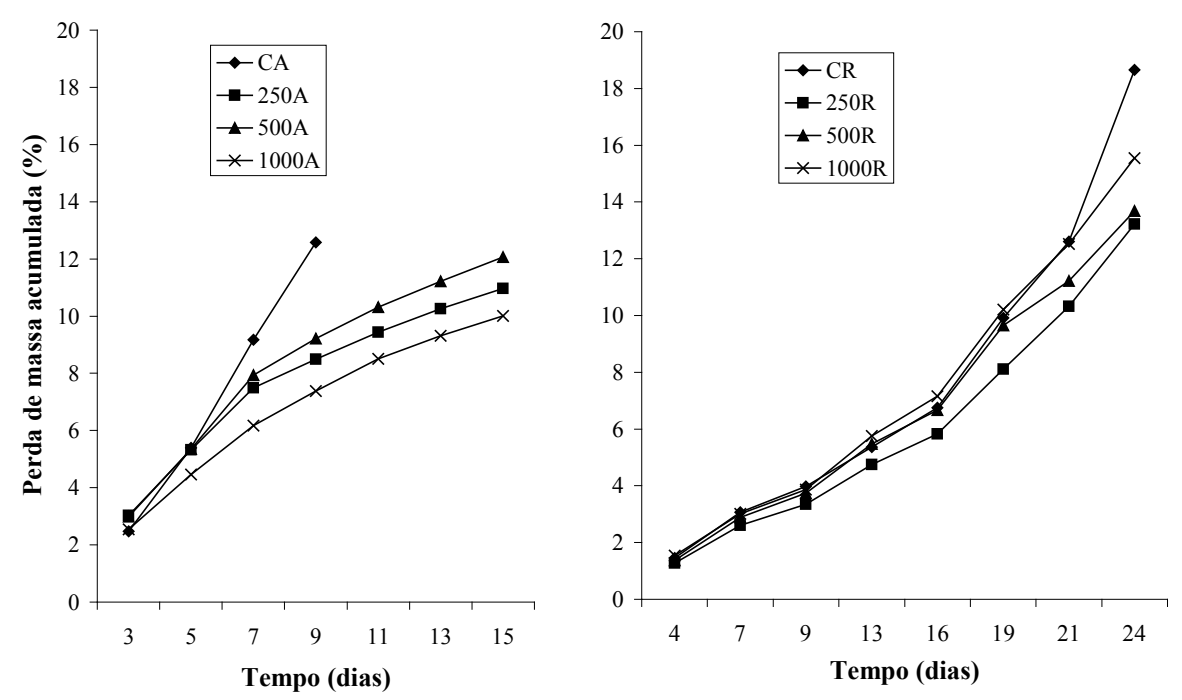

FIGURA 1 - Médias das perdas de massa de mangabas submetidas à aplicações de 1-MCP nas concentrações de 250; 500 e $1.000 \eta \mathrm{L} \mathrm{L}^{-1}$, durante $12 \mathrm{~h}$ e armazenadas em condição ambiente $\left(27 \pm 3^{\circ}\right.$ C e $45 \pm 10 \%$ UR): Controle (CA), (250A), (500A) e (1.000A) ou sob refrigeração $\left(11 \pm 1{ }^{\circ} \mathrm{C}\right.$ e $75 \pm 10 \%)$ : Controle (CR), (250R), (500R) e (1.000R).

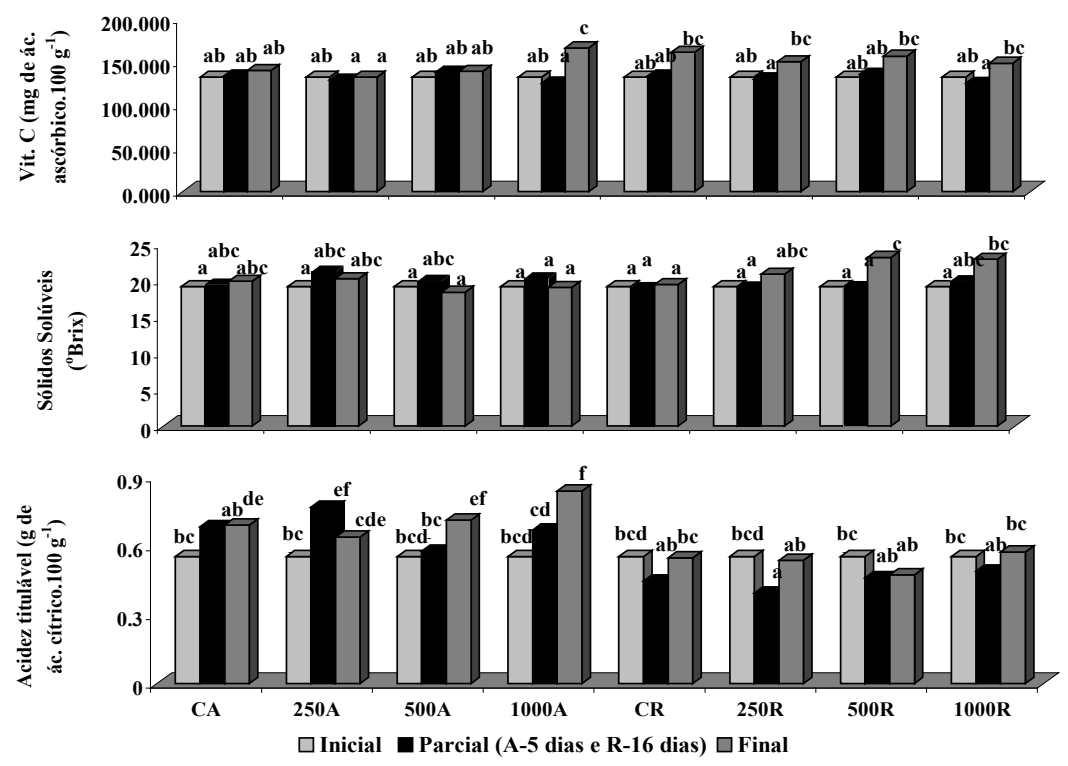

FIGURA 2 - Médias de características químicas de mangabas submetidas à aplicações de 1-MCP nas concentrações de 250; 500 e $1.000 \eta \mathrm{L} \mathrm{L}^{-1}$, durante $12 \mathrm{~h}$, armazenadas em condição ambiente (27士 $3^{\circ} \mathrm{C}$ e $45 \pm 10 \%$ UR): Controle (CA), (250A), (500A) e (1.000A) ou sob refrigeração (11 \pm $1{ }^{\circ} \mathrm{C}$ e $\left.75 \pm 10 \%\right)$ : Controle (CR), (250R), (500R) e (1.000R). 


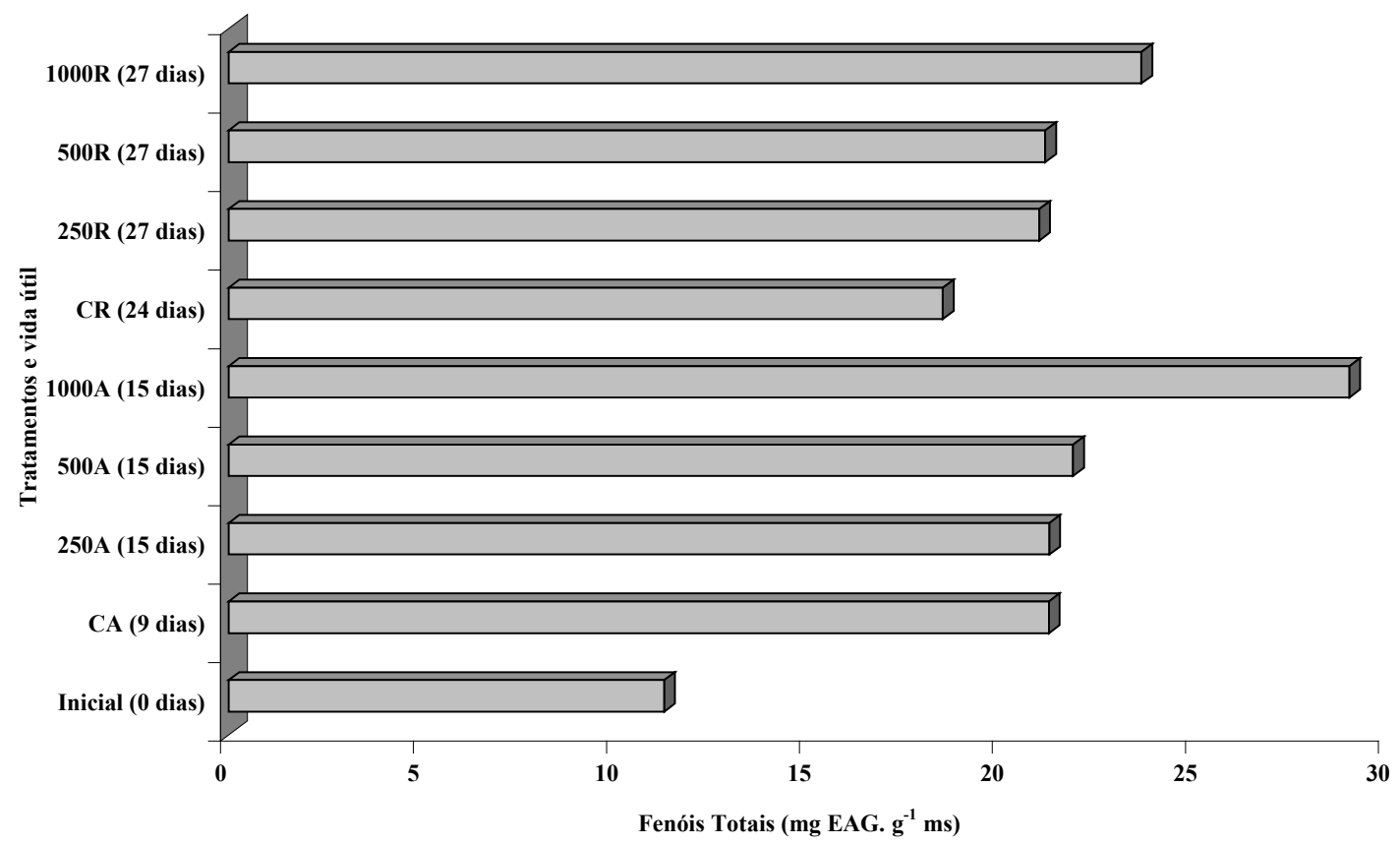

FIGURA 3 - Valores de fenóis totais no início e ao final do armazenamento de mangabas submetidas à aplicações de 1-MCP nas concentrações de 250; 500 e $1.000 \eta \mathrm{L} \mathrm{L}^{-1}$, durante $12 \mathrm{~h}$, armazenadas em condição ambiente $\left(27 \pm 3^{\circ} \mathrm{C}\right.$ e $45 \pm 10 \%$ UR): Controle (CA), (250A), (500A) e (1.000A) ou sob refrigeração $\left(11 \pm 1^{\circ} \mathrm{C}\right.$ e $\left.75 \pm 10 \%\right)$ : Controle (CR), (250R), (500R) e (1.000R).

\section{CONCLUSÕES}

1 - O armazenamento dos frutos a $10^{\circ} \mathrm{C}$ favorece o prolongamento da sua vida útil, com menor perda de massa, manutenção dos teores de acidez titulável, sólidos solúveis e vitamina $\mathrm{C}$; e quando associado à aplicação de 1-MCP, reduz os descartes por amadurecimento excessivo.

2 - O uso de 1-MCP em mangabas, independentemente das três concentrações utilizadas, seguida de armazenamento em temperatura ambiente, apresenta grande benefício na conservação pós-colheita dos frutos, com aumento da vida útil e da acidez titulável e redução da perda de massa.

\section{AGRADECIMENTOS}

Agradecemos à Fundação de Apoio ao Desenvolvimento do Ensino, Ciência e Tecnologia do Estado de Mato Grosso do Sul e ao Conselho Nacional de Desenvolvimento Científico e Tecnológico, pelo apoio financeiro, e à Rohm and Haas Química Ltda., pela disponibilização do Smart Fresh.

\section{REFERÊNCIAS}

ALMEIDA, M. M. B.; SOUSA, P. H. M.; ARRIAGA, A. M. C.; PRADO, G. M.; MAGALHÃES, C. E. C.; MAIA, G. A.; LEMOS, T. L. G. Bioactive compounds and antioxidant activity of fresh exotic fruits from northeastern Brazil, Food Research International, Darking, v.44, n.7, p.2155-2149, 2011.

ALVES, R. E.; FILGUEIRAS, H. A. C.; MOURA, C. F. H. Caracterização de frutas nativas da América Latina. Jaboticabal: Funep, 2000. 66 p. (Série frutas nativas, 9).

AYRES, M.; AYRES J.R., M.; AYRES, D. L.; SANTOS, A. de A. S. Aplicações estatísticas nas áreas das ciência biomédicas (BioEstat 5.0). Belém: Sociedade Civil Mamirauá, 2007. 324p.

BRASIL. Ministério da Saúde. Agência Nacional de Vigilância Sanitária. Métodos físico-químicos para análises de alimentos. Brasília: Ministério da Saúde, 2005.1018p. 
CANUTO, G. A. B.; XAVIER, A. A. O.; NEVES, L. C.; BENASSI, M. de T. Caracterização físico-química de polpa de frutos da Amazônia e sua correlação com a atividade antirradical livre. Revista Brasileira de Fruticultura, Jaboticabal, v. 32, n. 4, p. 1196-1205, 2010.

CARNELOSSI, M. A. G.; TOLEDO, W. F. F.; SOUZA, D. C. L.; LIRA, M. DE L.; SILVA, G. F. DA; JALALI, V. R.R.; VIÉGAS, P. R. A. Conservação pós-colheita de mangaba (Hancornia speciosa Gomes). Ciência e Agrotecnologia., Lavras, v. 28, n. 5, p. 1119-1125, 2004.

CARVALHO, C. R. L.; MANTOVANI, D. M. B.; CARVALHO, P. R. N.; MORAES, R. M. Análises químicas de alimentos. Campinas: Instituto de Tecnologia de Alimentos, 1990. 120 p. (Manual técnico.)

CHITARRA, M. I. F.; CHITARRA, A. B. Pós-colheita de frutos e hortaliças: fisiologia e manuseio. 2. ed. rev. e ampl. Lavras: UFLA, 2005.

DOLLHOJO, E. T.; ABREU, C.M.P. DE; ASMAR, S. A.; HOJO, R. H.; CÔRREA, A. D.; VILAS BOAS, E. V. de B. Avaliação da qualidade de manga 'palmer' tratada com 1-metilciclopropeno e armazenada sob refrigeração e condição ambiente. Revista Brasileira de Fruticultura, Jaboticabal, v. 31， n. 1, 2009.

EMPRAPA. Sistema de produção da mangaba para os tabuleiros costeiros e baixadas litorâneas. Versão eletrônica, nov/2007. Disponível em: $<$ http://sistemasdeproducao.cnptia.embrapa.br/ FontesHTML/Mangaba/SistemaProducaoMangabaTabuleirosCosteiros/Introducao.html>. Acesso em: jun. 2010.

FERREIRA, E. G.; MARINHO, S.J.O. Produção de frutos da mangabeira para consumo in natura e industrialização. Tecnologia e Ciência Agropecuária, João Pessoa, v.1, n.1, p.9-14, 2007.

GUILHERME, D. O.; SANTOS, A. M.; PAULA, T. O. M. DE; ARAUJO, C. B.; SANTOS, W. G.; SILMA LEITE ROCHA, S. L.; CALDEIRA JUNIOR, C. F.; MARTINS, E. R. Ecogeografia e etnobotânica da mangaba (Hancornia speciosa) no norte de Minas Gerais. Revista Brasileira de Biociências, Porto Alegre, v. 5, supl. 1, p. 414-416, 2007.

IBGE. Produção extrativa vegetal. Disponível em: <http://www.sidra.ibge.gov.br>. Acesso em: jul. 2010.
LORENZI, H. Árvores brasileiras: manual de identificação e cultivo de plantas arbóreas nativas do Brasil. Nova Odessa: Instituto Plantarum, 2009. Disponível em: $<$ http://www.clubedasemente.org.br/ mangabeira.html>. Acesso em: jun. 2010.

MARIN, A. M. F.; SIQUEIRA, E. M. A.; ARRUDA, S. F. Minerals, phytic acid and tannin contents of 18 fruits from the Brazilian savanna. International Journal of Food Sciences and Nutrition, Abingdon, v. 60, n.7, p. 180-190, 2009.

MOURA, C. F. H.; ALVES, R. E.; FILGUEIRAS, H. A. C.; ARAÚJO, N. C. C.; ALMEIDA, A. S. Quality of fruits native to latin america for processing: mangaba (Hancornia speciosa Gomes). Acta Horticulturae, The Hague, v. 2, n. 575, p. 549-554, 2002

ROESLER, R.; MALTA, L.G.; CARRASCO, L.C.; HOLANDA, R.B.; SOUZA, C.A.S.; PASTORE, G.M. Atividade antioxidante de frutas do cerrado. Ciência e Tecnologia de Alimentos, Campinas, v. 27, n. 1, p. 53-60, 2007.

RUFINO, M.S.M.; ALVES, R.E.; BRITO, E. S.; PÉREZ-JIMÉNEZ, J.; SAURA-CALIXTO, F.; MANCINI-FILHO, J. Bioactive compounds and antioxidant capacities of 18 non-traditional tropical fruits from Brazil. Food Chemistry, London, v.121, p. 996-1002, 2010.

SANTOS, A. F. dos; SILVA, S.de M.; MENDONÇA, R. M. N.; ALVES, R. E. Conservação pós-colheita de mangaba em função da maturação, atmosfera e temperatura de armazenamento. Ciência e Tecnologia de Alimentos, Campinas, v. 29, n. 1, p. 85-91, 2009.

SILVA, M. R.; LACERDA, D. B. C. L.; SANTOS, G. G. S.; MARTINS, D. M. de O. Caracterização química de frutos nativos do cerrado. Ciência Rural, Santa Maria, v.38, n.6, p.1790-1793, 2008.

SOARES, F. P.; PAIVA, R.; NOGUEIRA, R. C.; OLIVEIRA, L. M. de; SILVA, D. R. G.; PAIVA, P. D. de O. Cultura da mangabeira. Boletim Agropecuário, Lavras, n.67, p.1-12, 2005.

SOUZA, F. G. de; FIGUEIREDO, R. W de; ALVES, R. E.; MAIA, G. A.; ARAÚJO, I. A. de; Qualidade pós-colheita de frutos de diferentes clones de mangabeira (Hancornia speciosa GOMES). Ciência e Agrotecnologia, Lavras, v. 31, n. 5, p. 1449-1454, 2007. 
SOUZA,C.S.; SILVA,S.A.; COSTA, M.A.P.C.; DANTAS, A.C.V.L.; COSTA, C.A.L.C.; ALMEIDA,W.A.B.; PEIXOTO,C.P. Mangaba: perspectivas e potencialidades. Bahia Agrícola, Brasília, v.7, n.1, p.9-14, 2007.

VASCO, C.; RUALES, J.; KAMAL-ELDIN, A. Total phenolic compounds and antioxidant capacities of major fruits from Ecuador. Food Chemistry, London, v. 111, n. 4, p. 816-823, 2008.
SWAIN, T.; HILLS, W.E. The phenolics constituents of prumus domestica: the quantitative analysis of phenolic constituents. Journal of the Science Food and Agriculture, London, v. 10, n. 1, p. 63-68, 1959. 